\title{
Incidencia del modelo Kano en la rentabilidad de las Mypes de la ciudad de Huancayo
}

\section{Incidence of the Kano model in the profitability of the Mypes of Huancayo city}

'Munguía Palacios, R.V.; García Puente, D.J.

Facultad de Contabilidad, Universidad Nacional del Centro del Perú

Email: rmunguia@uncp.edu.pe

\section{Resumen}

De acuerdo a la Estructura Empresarial 2015 del INEI, al 31 de diciembre del 2015, en nuestro país existen 2,042,992 unidades empresariales, de los cuales las micro y pequeñas representa el $94.6 \%$ y el $4.4 \%$ respectivamente y que, en conjunto, la Mype constituye el $99.0 \%$ del total de las empresas; mientras que la tasa de crecimiento y defunción es del $12.3 \%$ y $8.0 \%$ respectivamente y que la ciudad de Huancayo tiene estas mismas características.

Por lo que, atento a su vigencia en el mercado, se considera importante estudiar el modelo Kano de satisfacción de clientes, aspecto clave para el éxito; por esta razón, se ha investigado esta variable, teniendo presente que la satisfacción proviene del cliente, quien al final juzga el producto y/o servicio y toma la decisión de compra.

Para el estudio, se utilizó el método de casos, cuyos resultados nos permite inferir a las Mypes de la ciudad de Huancayo en el rubro de alimentos y, se considera que la satisfacción del cliente, es un elemento que todo empresario debe privilegiar y orientar a encarar el tema en su empresa; lo que ha permitido contrastar la hipótesis, llegando a la conclusión de la incidencia moderada de la satisfacción del cliente en la variable rentabilidad, debido a que el concepto de rentabilidad tiene otras dimensiones, aparte del nivel de ventas, como el precio, costos e impuesto, entre otros, tal como lo refiere el sistema Dupont y que en el contexto actual, a las empresas les preocupa sostener sus niveles de ingreso y que esta se traduzca en rentabilidad.

Palabras Clave: satisfacción, rentabilidad, modelo Kano, Mypes

\begin{abstract}
According to the 2015 Business Structure of INEI, up to December 31, 2015 in our country, there are $2,042,992$ business units, of which the micro and small represents $94.6 \%$ and $4.4 \%$ respectively and together Mype constitutes $99.0 \%$ of total companies; while the rate of growth and death is $12.3 \%$ and 8.0 $\%$ respectively and our Huancayo city has these same characteristics.

So, regarding its market validity, studying the kano model of customer satisfaction is considered important, key aspect for success; for this reason this variable has been investigated, bearing in mind that satisfaction comes from the client who ultimately judges the product and / or service and makes his purchase decision.

For the study, the case method was used, the results of which allow us to infer the Mypes of the Huancayo city in the food category and we consider that customer satisfaction is an element that every entrepreneur must privilege and guide their company to face the topic; which has allowed us to test the hypothesis, reaching the conclusion of the moderate incidence of customer satisfaction in the profitability variable, because the profitability concept has other dimensions, apart from the level of sales, such as price, costs and tax among other, as referred by the Dupont system and that in the current context companies are concerned about sustaining their income levels and that these are translated into profitability.
\end{abstract}

Keywords: satisfaction, profitability, Kano model, Mypes. 


\section{Introducción}

Al 31 de diciembre del 2015, de acuerdo al INEI, las Mypes en el Perú representan el 99.0 \% y la tasa de defunción es del $8.0 \%$ y, en la ciudad de Huancayo se replica estas mismas características, por lo que es importante coadyuvar al sostenimiento y crecimiento de estas unidades económicas y evitar la desaparición; además, otra de las particularidades está representada por su carácter familiar; por estas razones se ha considerado importante investigar el concepto de satisfacción de cliente, el cual se ha esclarecido con la aplicación del modelo propuesto y aplicado en otras realidades por el Dr. Noriaki Kano, conocido en el mundo académico y empresarial como "modelo Kano"; quien plantea los diferentes niveles de calidad, el cual es clasificado en motivante, deseada, esperada e indiferente, también, es necesario encuadrar los atributos del bien y/o servicio y, que al ofrecerlos al cliente, es preciso observar la actitud, frente a estas características diferenciadores del producto y, como esta, orienta la atención e influencia en la decisión de compra.

Se entiende que la satisfacción proviene del cliente y no es la percepción de la empresa, porque al final es quien juzga el bien y/o servicio y en base a ello decide libremente, sin presión alguna, la compra y la empresa quiere que se decida por su producto; para ello, se debe orientar a la unidad económica, a enfocarse en ese factor tan crucial en este contexto y que su permanencia es importante, del que no se puede esperar que se produzcan quejas para recién reaccionar, sino por el contrario, estar siempre atentos y alertas al comportamiento dentro de la organización.

El presente trabajo de investigación, se justifica por su contribución en el sostenimiento de estas unidades económicas, consideradas como Mype, debido a su trascendencia en el contexto económico nacional, cuya vigencia y aporte en la creación de la mano de obra es importante, teniendo en cuenta al factor cliente y, en él, un elemento relevante es el logro de satisfacción, por lo que se contribuye a su permanencia en el mercado y se entiende que esto es posible, siempre en cuando la empresa obtenga rentabilidades sostenibles en el tiempo.

$\mathrm{Al}$ analizar la incidencia de la variable independiente, satisfacción de cliente y; en la variable dependiente, rentabilidad, se ha estudiado los conceptos y dimensiones de tal manera que ha servido para contrastar la hipótesis de trabajo, cumpliendo de esta manera los objetivos inicialmente planteados en el proyecto de investigación.

\section{Materiales y métodos}

\section{Metodología de investigación}

Entendiendo a la metodología como los procedimientos y técnicas que debemos de utilizar para el desarro- llo del trabajo; se ha utilizado en su desarrollo; el método deductivo e inductivo, en la cual se ha estudiado de manera general todos los datos de las variables de la investigación, lo que ha permitido llegar a conclusiones particulares; luego se ha hecho uso del método analítico, el cual ha permitido analizar e interpretar los diferentes resultados de la encuesta realizada. Por otro lado, se ha utilizado el método de casos el cual permite estudiar los aspectos generales y particulares de una empresa cuyos resultados han inferido en las demás empresas Mypes de la ciudad de Huancayo en el rubro de alimentos.

\section{Tipo de investigación}

La investigación efectuada es explicativa, debido a que trata de explicar la incidencia de la variable independiente, satisfacción del cliente, en la variable dependiente, rentabilidad; para ello, se ha utilizado el método formulado por el Dr. Kano, con la finalidad de establecer con claridad el concepto de calidad y su relación son la satisfacción del cliente el cual de alguna forma incide en la estabilización e incremento de las ventas de las empresas y por ende su rentabilidad.

\section{Alcance de la investigación}

La investigación es mixta porque contiene aspectos cualitativos como cuantitativos en la cual, al ser explicativo, explica cómo una variable incide en la otra.

\section{Población y muestra}

Considerando a la población como el conjunto de personas, cosas y otros de los que se pretende conseguir algo; en el caso de la presente investigación, es necesario definir su objeto de estudio; lo que se procura en el presente trabajo, es determinar el nivel de satisfacción de los clientes, para ello, se emplea el modelo Kano y, luego, se relaciona el nivel de satisfacción con la rentabilidad, el mismo que será analizado en la empresa modelo, considerada como Mype, de la ciudad de Huancayo.

Para este estudio se desarrolló la investigación en una empresa Mype de la ciudad de Huancayo - por la disponibilidad de la información- en el rubro de sándwiches, en la cual la población fue el número de clientes que concurren en forma diaria y, a partir de ello, se ha deducido la muestra representativa de la población a investigar y sus resultados permitirán inferir a las demás empresas mypes de este rubro.

\section{Población}

La población está conformada por los clientes de la empresa, en el rubro de sándwiches, en la cual se determinará el nivel de satisfacción de los clientes empleando el modelo Kano. Por lo que el objeto de estudio para este trabajo de investigación, está constituido por los clientes en el rubro de sándwiches de la empresa de nombre comercial "Huaychulo". 
El objeto de análisis, es una pequeña empresa, cuya razón social es Minimarket La China E.I.R.L., con Ruc No 20601117542, cuyo domicilio fiscal es el Jirón Arequipa $\mathrm{N}^{\circ} 454$ de la ciudad de Huancayo e inscrita en el Registro Mype del Ministerio de Trabajo y Promoción Social, dedicada a la comercialización de productos alimenticios, para la cual se ha dividido en las áreas de carnes, embutidos, lácteos, preparados, panes, restaurant y sándwiches; productos producidos por la empresa Alimentos Josecito EIRL, en la cual se producen los embutidos en sus diversas variedades; panes de hamburguesa y los panes artesanales denominadas "Chupaca" en sus variedades de bollos, con o sin manteca y misti; además, de la papa picada.

En el área de sándwiches, la empresa tiene una lista de productos, los que son ofrecidos al paso y en mesa del salón del segundo piso de su local; para ello, tiene una carta exclusiva para esta atención. Los productos que ofrecen se clasifican los sándwiches en hamburguesas de res y de pollo básicos y con sus combinaciones de huevo, queso Edam, jamón inglés, tocino ahumado, palta, camote, entre otros adicionales; luego, se tiene a los sándwiches de hot dog, chorizo, de pollo desmenuzado de jamones, con sus diferentes combinaciones de huevo, queso edam, jamón inglés, tocino ahumado o cualquier otro producto que el cliente solicite y; en el lado derecho de la lista de precios, se cuenta con productos que se expenden en platos descartables, como son las salchipapas con sus diferentes combinaciones, destacándose en ello las combinaciones de las salchichas; el hot tradicional, el picante, la Viena y el Frankfurt, la papa amarilla en vez de la papa pericholi, que es la que se usa comunmente.

La población, objeto de análisis, son los clientes de la barra, quienes adquieren sus productos de la lista de precios descrito en el cuadro anterior, para ello identificamos a la población que generalmente es gente joven que se encuentra en una edad de 18 a 35 años entre varones y mujeres $y$, para establecer el número de personas con estas características, hemos obtenido información del sistema de caja de la empresa; en la cual se registran un promedio diario de 400 clientes, en base a ello, se determinó la muestra.

\section{Muestra}

Considerando que la muestra es una pequeña parte que representa a la población, se ha clasificado a los clientes en el área de sándwiches al paso, quienes generalmente tienen una característica en común; gente joven, entre varones y mujeres que fluctúan en edades de 18 a 35 años.

Para la determinación del valor de n0, se considera el valor de confianza (z) de $95 \%$; mientras que el margen de error (e) es del $5 \%$ y para la probabilidad de éxito
(P) se considera el valor de 0.5; con ello, se estableció el valor de la probabilidad de fracaso (q), planteando la ecuación $(P+q=1)$ y si $P=0.5$, entonces se despeja el valor de q, que en este caso sería 0.5. Los datos que se analizaron arriba se resumen en la Tabla 1.

Tabla 1

Datos para la determinación del valor de $\mathrm{n}_{0}$

\begin{tabular}{ccc}
\hline $\mathbf{n}$ & Tamaño de la muestra & $?$ \\
\hline N & Población & 400 \\
z & Nivel de confianza & 1.96 \\
e & error muestral & 0.05 \\
P & Probabilidad de éxito & 0.5 \\
q & Probabilidad de fracaso & 0.5 \\
\hline
\end{tabular}

Con estos datos se aplicó la fórmula del muestreo aleatorio simple, con el objeto de determinar el valor de no, de esta forma se conoció el tamaño de la muestra.

Desarrollando se tiene:

$$
\begin{gathered}
n_{0}=\frac{Z^{2} \times P \times q}{e^{2}}=\frac{1.96^{2} \times 0.5 \times 0.5}{0.05^{2}}=384.16 \\
n_{0}=\mathbf{3 8 4}
\end{gathered}
$$

El valor de no, resulta 384, que vendría a ser el tamaño de la muestra, pero como se conoce la población, se procedió a ajustar la fórmula para determinar la muestra, el cual es el siguiente:

$$
n_{1}=\frac{n_{0}}{1+\frac{\left(n_{0}-1\right)}{N}}=\frac{384}{1+\frac{384-1}{400}}=196
$$

De acuerdo a este cálculo, se ha determinado que el tamaño de la muestra es de 196 clientes a la que se aplicó la encuesta, con la finalidad de establecer su grado de satisfacción.

\section{Resultados}

\section{Estudio sociodemográfico del cliente}

\section{Género}

En la Tabla 2, se muestra a los clientes entrevistados, según género.

Tabla 2

Clientes por género

\begin{tabular}{ccc}
\hline Genero & $\mathbf{N}^{\mathbf{0}}$ clientes & $\mathbf{\%}$ \\
\hline Mujer & 110 & 56.1 \\
Varon & 86 & 43.9 \\
Total & 196 & 100.0 \\
\hline
\end{tabular}

La información contenida en la tabla anterior, se puede apreciar en la Figura 1. 
Figura 1

Clientes por género

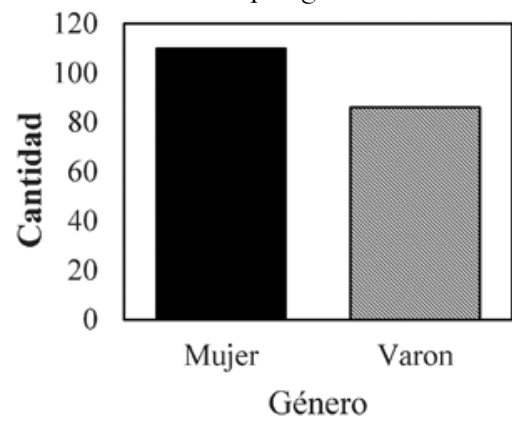

La muestra analizada, está compuesta por el $56.1 \%$ de clientes mujeres y $43.9 \%$ de varones, observándose que una ligera mayoría son clientes mujeres.

\section{Estado civil}

La información obtenida, referente a este rubro, se presenta en la Tabla 3.

Tabla 3

Estado civil de los clientes

\begin{tabular}{lcc}
\hline Estado civil & $\mathbf{N}^{\mathbf{0}}$ clientes & \% \\
\hline Soltero & 116 & 59.2 \\
Casado & 40 & 20.4 \\
Conviviente & 28 & 14.3 \\
No responde & 12 & 6.1 \\
$\quad$ TOTAL & $\mathbf{1 9 6}$ & $\mathbf{1 0 0 . 0}$ \\
\hline
\end{tabular}

Los clientes del área de sándwiches de la tienda, de acuerdo a la encuesta realizada, refieren que el $59.2 \%$ son solteros; mientras que el $20.4 \%$ son casados y el $14.3 \%$ es conviviente; la misma que se puede observar en la Figura 2.

Figura 2

Estado civil de los clientes

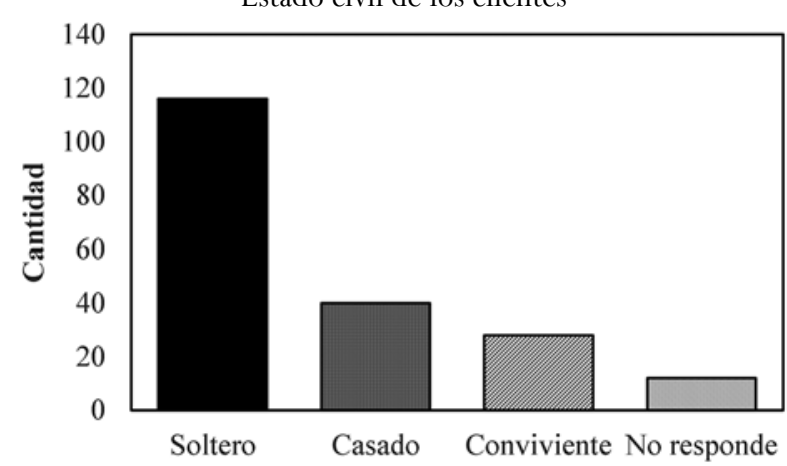

\section{Edad}

La edad de los clientes generalmente fluctúa entre los 18 a 21 años, el cual representa el $41.8 \%$; mientras que la edad de los clientes en el rango de 26 a 29 años representa el $23.0 \%$; seguida por el rango de 22 a 25 años con $17.9 \%$; mientras que el rango de 34 a 37 años represen- ta el $9.7 \%$ y; del rango del 30 a 33 años, los representa el $7.7 \%$. lo que se aprecia en la Tabla 4.

Tabla 4

Edad de los clientes

\begin{tabular}{ccc}
\hline Rango & $\mathbf{N}^{\mathbf{0}}$ clientes & $\mathbf{\%}$ \\
\hline $18-21$ & 82 & 41.8 \\
\hline $22-25$ & 35 & 17.9 \\
\hline $26-29$ & 45 & 23.0 \\
\hline $30-33$ & 15 & 7.7 \\
\hline $34-37$ & 19 & 9.7
\end{tabular}

Y con el objeto de apreciar estas características, presentamos la Figura 3.

Figura 3

Edad de los clientes

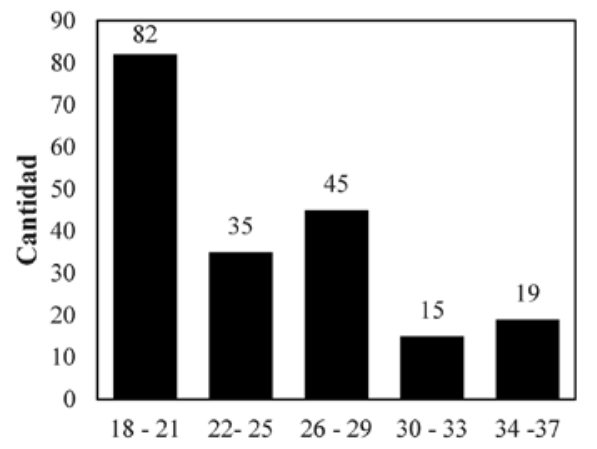

\section{Nivel de educación}

Sobre este aspecto, se ha efectuado la pregunta sobre el nivel educativo de los encuestados, lo que ha señalado que el $68 \%$ son estudiantes de nivel superior; mientras que el $32 \%$ se consideraran como estudiantes del nivel secundario, tal como se ve en la Figura 4.

\section{Figura 4}

Nivel de educación de los clientes

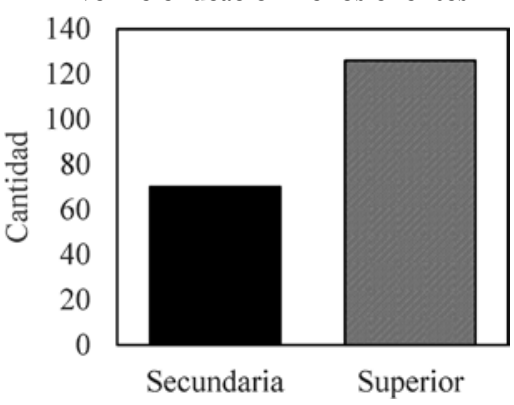

Expectativas de los clientes que adquieren sus productos en los ambientes de comida

Con la finalidad de conocer las expectativas de los clientes que adquieren productos en la zona de comida, se elaboró una encuesta, la que fue dirigida a la muestra de 196 clientes, las que previamente se han analizado en el acápite 3.1 (Tabla 5). 
Tabla 5

Diferentes dimensiones que engloba las características que el cliente busca en todo ambiente de comida

\begin{tabular}{|c|c|c|c|c|c|c|c|}
\hline \multirow{3}{*}{ Dimension } & \multirow{3}{*}{ Atributo } & \multicolumn{4}{|c|}{ Calidad modelo Kano } & \multirow{3}{*}{$\mathbf{n}$} & \multirow{3}{*}{$\%$} \\
\hline & & Motivante & Deseada & Esperada & Indiferente & & \\
\hline & & Atrayente & Basico & Rechazo & Indiferencia & & \\
\hline \multirow{4}{*}{ Calidad } & Presentación del local & 69 & 95 & 22 & 10 & 196 & 100.0 \\
\hline & $\begin{array}{l}\text { Ingredientes de los } \\
\text { Sandwiches }\end{array}$ & 25 & 137 & 12 & 22 & 196 & 100.0 \\
\hline & Sabor del sandwiches & 26 & 138 & 11 & 21 & 196 & 100.0 \\
\hline & $\begin{array}{l}\text { Presentación del sand- } \\
\text { wichs }\end{array}$ & 15 & 130 & 12 & 39 & 196 & 100.0 \\
\hline \multirow{3}{*}{ Variedad } & Productos de la carta & 69 & 105 & 12 & 10 & 196 & 100.0 \\
\hline & Variedad de cremas & 79 & 95 & 10 & 12 & 196 & 100.0 \\
\hline & Variedad de ensaladas & 78 & 101 & 9 & 8 & 196 & 100.0 \\
\hline Precio & Relación calidad - precio & 52 & 122 & 10 & 12 & 196 & 100.0 \\
\hline \multirow{3}{*}{$\begin{array}{l}\text { Promoción } \\
\text { del producto }\end{array}$} & $\begin{array}{l}\text { Existencia de promo- } \\
\text { ciones }\end{array}$ & 71 & 100 & 10 & 15 & 196 & 100.0 \\
\hline & $\begin{array}{l}\text { Frecuencia de las pro- } \\
\text { mociones }\end{array}$ & 59 & 120 & 5 & 12 & 196 & 100.0 \\
\hline & $\begin{array}{l}\text { Variedad de las promo- } \\
\text { ciones }\end{array}$ & 62 & 118 & 6 & 10 & 196 & 100.0 \\
\hline Rapidez & $\begin{array}{l}\text { Tiempo de espera del } \\
\text { cliente }\end{array}$ & 112 & 26 & 50 & 8 & 196 & 100.0 \\
\hline \multirow{3}{*}{$\begin{array}{l}\text { Amabilidad } \\
\text { y atención }\end{array}$} & Saludo de los empleados & 65 & 110 & 7 & 14 & 196 & 100.0 \\
\hline & $\begin{array}{l}\text { Empatía con los em- } \\
\text { pleados }\end{array}$ & 57 & 118 & 8 & 13 & 196 & 100.0 \\
\hline & $\begin{array}{l}\text { Gentileza de los em- } \\
\text { pleados }\end{array}$ & 72 & 110 & 5 & 9 & 196 & 100.0 \\
\hline \multirow{3}{*}{ Información } & $\begin{array}{l}\text { Conocimiento de los } \\
\text { empleados en relación al } \\
\text { producto }\end{array}$ & 52 & 99 & 12 & 33 & 196 & 100.0 \\
\hline & $\begin{array}{l}\text { Descripción de los pro- } \\
\text { ductos de la carta }\end{array}$ & 48 & 109 & 15 & 24 & 196 & 100.0 \\
\hline & $\begin{array}{l}\text { Rapidez en la entrega de } \\
\text { la información }\end{array}$ & 59 & 115 & 10 & 12 & 196 & 100.0 \\
\hline \multirow{3}{*}{$\begin{array}{l}\text { Ambiente y } \\
\text { decoración }\end{array}$} & Música agradable & 75 & 112 & 4 & 5 & 196 & 100.0 \\
\hline & Olores agradables & 65 & 118 & 6 & 7 & 196 & 100.0 \\
\hline & Colores agradables & 72 & 114 & 8 & 2 & 196 & 100.0 \\
\hline \multirow{6}{*}{ Higiene } & $\begin{array}{l}\text { Pisos limpios e higié- } \\
\text { nicos }\end{array}$ & 92 & 86 & 3 & 15 & 196 & 100.0 \\
\hline & $\begin{array}{l}\text { Barras limpias e higié- } \\
\text { nicas }\end{array}$ & 87 & 96 & 2 & 11 & 196 & 100.0 \\
\hline & $\begin{array}{l}\text { Zona de atención cómo- } \\
\text { da y amplia }\end{array}$ & 93 & 89 & 4 & 10 & 196 & 100.0 \\
\hline & $\begin{array}{l}\text { Presentación personal } \\
\text { de aseo }\end{array}$ & 95 & 82 & 3 & 16 & 196 & 100.0 \\
\hline & $\begin{array}{l}\text { Tazones limpios e hi- } \\
\text { giénicos }\end{array}$ & 104 & 87 & 2 & 3 & 196 & 100.0 \\
\hline & $\begin{array}{l}\text { Contenedor de desper- } \\
\text { dicios }\end{array}$ & 101 & 85 & 2 & 8 & 196 & 100.0 \\
\hline
\end{tabular}

Fuente: elaboración propia 
Para ello, se ha empleado el modelo Kano, el cual ha permitido clasificar en las cuatro categorías de la calidad:

- Calidad motivante; este tipo de calidad se ha denominado "Atrayente" y, que de acuerdo a lo esbozado por Moriaki Kano, son los atributos que el cliente no espera y de encontrarse en el productos o servicio, motiva a que el cliente realice su compra y; de no estar incluido, no aumentó ni se produce el rechazo de parte del cliente.

- Calidad deseada; es lo que el cliente espera obtener como mínimo de un producto o un servicio y se ha denominado "Básico", debido a las características mínimas o básicas que debe tener el producto o el servicio y lo que se debe de tener en cuenta, es que de faltar algún atributo, esto va afectar en la aceptación del productos o servicio, produciéndose el rechazo.

- Calidad esperada; se debe tener presente los atributos del producto o servicio que el cliente espera obtener, que van a influir en la aceptación o rechazo del bien o servicio. Se ha definido cono "Rechazable", debido a que la existencia o ausencia de los atributos van a influir en el rechazo del bien o servicio.

- Calidad de indiferente; los mismos que no influyen ni positivamente, ni negativamente en la percepción de satisfacción del cliente. A ello, se le ha considerado como "Indiferente"

Para efectuar el estudio sobre la satisfacción del cliente, en primer término, se ha clasificado los atributos en los cuatro tipos de calidad considerados en el modelo Kano; para ello, se ha determinados las diferentes dimensiones que engloba las características que el cliente busca en todo ambiente de comida, las que se han considerado en la encuesta, cuyos resultados se observan en la tabla precedente.

En base a este cuadro resumen de resultados de la encuesta efectuada a la muestra, se analizará cada una de ellas.

\section{Dimensión: Calidad}

En esta dimensión, se ha considerado los atributos de presentación de local; ingredientes, sabor y presentación de los sándwiches, los que se considera importantes, por cuanto que el cliente aprecia en primer término, la presentación del local, aspecto importante por cuanto es la primera impresión que tiene una persona cuando visita a un local puede ser familiar o comercial; en segundo lugar, se tiene la impresión referente al producto o servicio que se ofrece, en tal sentido se considera como indispensable la combinación adecuada de los ingredientes, el sabor y la presentación del bien o servicio.

De acuerdo a los resultados de la encuesta, se tiene que el mayor peso de los clientes es considerar a las características básicas que debe tener los atributos analizados, seguido de la característica de atrayente que de alguna forma influye en la motivación a la compra del bien, como se puede apreciar en la Tabla 6.
Tabla 6

Cuadro resumen de respuestas de cliente sobre atributos de calidad en \%

\begin{tabular}{|c|c|c|c|c|c|}
\hline \multirow[b]{2}{*}{ Atributo } & \multicolumn{4}{|c|}{ Respuestas } & \multirow[b]{2}{*}{$\%$} \\
\hline & 莺 & 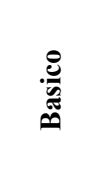 & 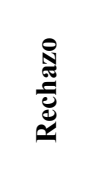 & 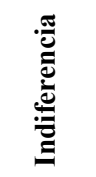 & \\
\hline $\begin{array}{l}\text { Presentación del } \\
\text { local }\end{array}$ & $35.2 \%$ & $48.5 \%$ & $11.2 \%$ & $5.1 \%$ & 100.0 \\
\hline $\begin{array}{l}\text { Ingredientes de los } \\
\text { sandwiches }\end{array}$ & $12.8 \%$ & $69.9 \%$ & $6.1 \%$ & $11.2 \%$ & 100.0 \\
\hline $\begin{array}{l}\text { Sabor del sand- } \\
\text { wiches }\end{array}$ & $13.3 \%$ & $70.4 \%$ & $5.6 \%$ & $10.7 \%$ & 100.0 \\
\hline $\begin{array}{l}\text { Presentación del } \\
\text { sandwichs }\end{array}$ & $7.7 \%$ & $66.3 \%$ & $6.1 \%$ & $19.9 \%$ & 100.0 \\
\hline
\end{tabular}

En la característica básica, encontramos que el $70 \%$ de los encuestados considera que el sabor es el factor determinante; mientras que lo atrayente está referida a la presentación de local con un 35.2\%; mientras que el de rechazo también está referida la presentación de local con $11.2 \%$ y; de la indiferencia, es la presentación de los sándwiches en $19.9 \%$. Estos resultados se contemplan apreciar en la Figura 5.

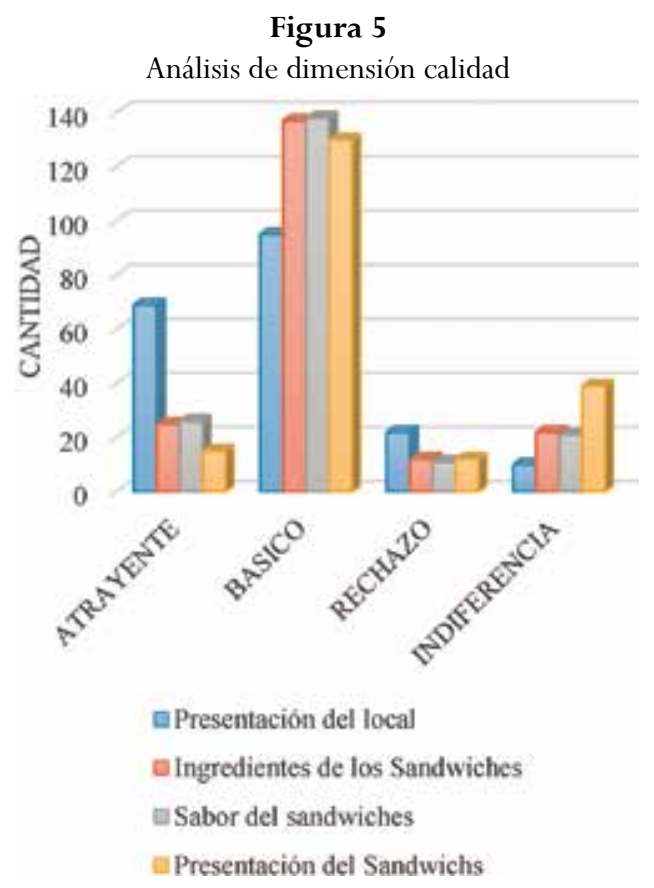

\section{Dimensión: Variedad}

En esta dimensión se han considerado los atributos a los productos de la carta; esto debido a la cantidad existente en el listado de la carta, da una sensación de variedad, el cual se puede lograr mediante las diferentes combinaciones que se pueden efectuar, de tal manera que se obtienen una variedad de productos que se pueden manejar adecuadamente y, de acuerdo a las respuestas, se observa 
que 105 clientes, de un total del 196, consideran como básico la variedad de productos que debe contener la carta para que puedan escoger libremente su producto.

El otro aspecto que se considera dentro de la disminución de variedad, está referida a las cremas y ensaladas, debido a que aporta el sabor y neutraliza el efecto de comida chatarra, puesto que la variedad de ensaladas que ofrece la empresa, de alguna forma contrarresta la campaña que se viene enfrentando en los últimos años con respecto al efecto nocivo para la salud del ser humano. En ello, se advierte que, 95 consideran a las cremas como básico y 101 como básico a la variedad de ensaladas.

En la Tabla 7, se analiza en términos porcentuales los atributos de variedad en la cual considera como básico el $53.6 \%$ a la variedad de productos ofrecidos en listado de productos: mientras que de lo atrayente consideran a la variedad de cremas en un $40.3 \%$ y de rechazo a los productos de la carta y de indiferencia a la variedad de cremas. Este análisis efectuado, se puede ver en la Figura 6 .

Tabla 7

Cuadro resumen de respuestas de cliente sobre atributos de variedad en $\%$

\begin{tabular}{|c|c|c|c|c|c|}
\hline \multirow[b]{2}{*}{ Atributo } & \multicolumn{4}{|c|}{ Respuestas } & \multirow[b]{2}{*}{$\%$} \\
\hline & 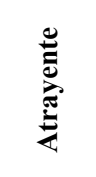 & 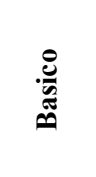 & 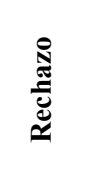 & 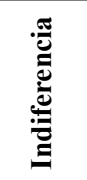 & \\
\hline $\begin{array}{l}\text { Productos de la } \\
\text { carta }\end{array}$ & $35.2 \%$ & $53.6 \%$ & $6.1 \%$ & $5.1 \%$ & 100.0 \\
\hline Variedad de cremas & $40.3 \%$ & $48.5 \%$ & $5.1 \%$ & $6.1 \%$ & 100.0 \\
\hline $\begin{array}{l}\text { Variedad de ensal- } \\
\text { adas }\end{array}$ & $39.8 \%$ & $51.5 \%$ & $4.6 \%$ & $4.1 \%$ & 100.0 \\
\hline
\end{tabular}

Figura 6

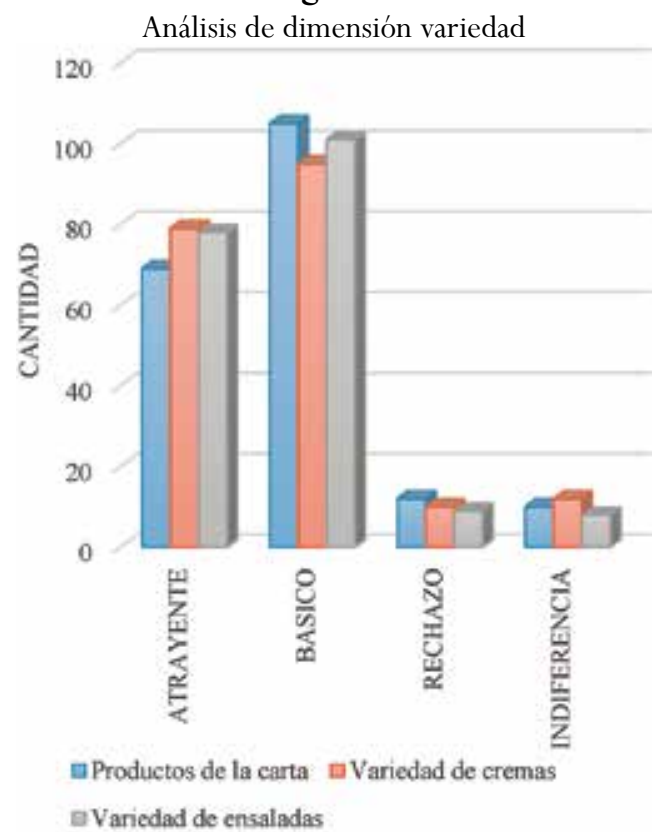

\section{Dimensión: Precio}

En esta dimensión, se considera el atributo de la relación calidad - precio, factor importante en el momento de tomar la decisión de compra y, de acuerdo a los resultados de la encuesta, se tiene que 122 clientes consideran como básico; mientras que 52 lo consideran atrayente.

En el análisis del atributo de la relación calidad precio, el $62.2 \%$ de los encuestados lo considera como básico; mientras que el $26.5 \%$, lo considera como atrayente; de rechazo el $5.1 \%$ y; el de indiferencia $6.1 \%$, como se muestra en la Tabla 8 . Los resultados del análisis efectuado se pueden observar en la Figura 7.

Tabla 8

Cuadro resumen de respuestas sobre atributos de precios - calidad en \%

\begin{tabular}{|c|c|c|c|c|c|}
\hline \multirow[b]{2}{*}{ Atributo } & \multicolumn{4}{|c|}{ Respuestas } & \multirow[b]{2}{*}{$\%$} \\
\hline & 节 & 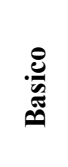 & 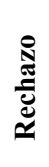 & 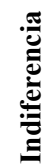 & \\
\hline $\begin{array}{l}\text { Relación calidad - } \\
\text { precio }\end{array}$ & 26.5 & 62.2 & 5.1 & 6.1 & 100 \\
\hline
\end{tabular}

Figura 7

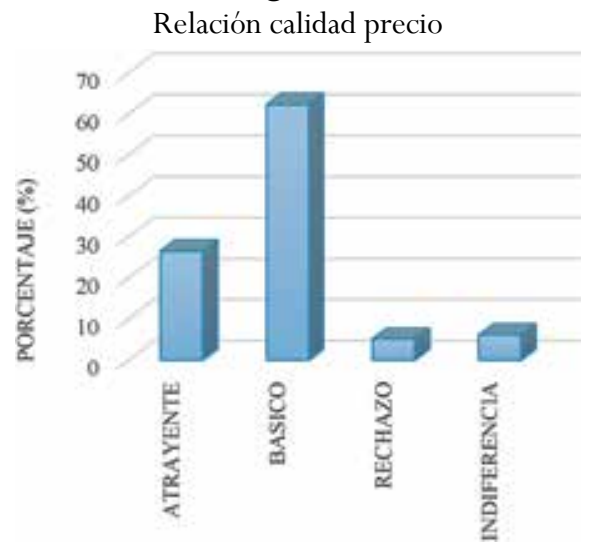

Dimensión: Promoción del producto

En cuanto a esta dimensión, está referida a las promociones que efectúa la empresa con la finalidad de hacer conocer a los clientes sobre nuevos productos, descuentos, adiciones de otros productos, entre otros aspectos. Dentro de esta dimensión se ha considerado los atributos de existencia, frecuencia y variedad de promociones y, de acuerdo a las respuestas de los entrevistados, consideran como básico 120 a la frecuencia de las promociones; mientras que la variedad de las promociones es considerada por 62 encuestados; mientras que el de rechazo son $10 \mathrm{y}$ de indiferencia a 15 .

En el cuadro de análisis de respuestas de estos atributos en términos porcentuales (Tabla 9), refiere que las 
frecuencias de las promociones son unos atributos básicos, pues el $61.2 \%$ lo considera de ese modo; mientras que el $36.2 \%$ lo considera como atrayente; y el $5.1 \%$ lo considera como rechazo y el $7.7 \%$ también lo considera de ese modo. Este análisis efectuado se encuentra en la Figura 8.

\section{Tabla 9}

Cuadro resumen de respuestas sobre atributos de promoción de producto

\begin{tabular}{|c|c|c|c|c|c|}
\hline \multirow[b]{2}{*}{ Atributo } & \multicolumn{4}{|c|}{ Respuestas } & \multirow[b]{2}{*}{$\%$} \\
\hline & 冚 & 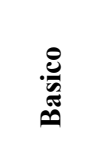 & 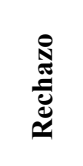 & 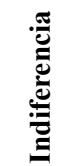 & \\
\hline $\begin{array}{l}\text { Existencia de pro- } \\
\text { mociones }\end{array}$ & $36.2 \%$ & $51.0 \%$ & $5.1 \%$ & $7.7 \%$ & 100 \\
\hline $\begin{array}{l}\text { Frecuencia de } \\
\text { promociones }\end{array}$ & $30.1 \%$ & $61.2 \%$ & $2.6 \%$ & $6.1 \%$ & 100 \\
\hline $\begin{array}{l}\text { Variedad de promo- } \\
\text { ciones }\end{array}$ & $31.6 \%$ & $60.2 \%$ & $3.1 \%$ & $5.1 \%$ & 100 \\
\hline
\end{tabular}

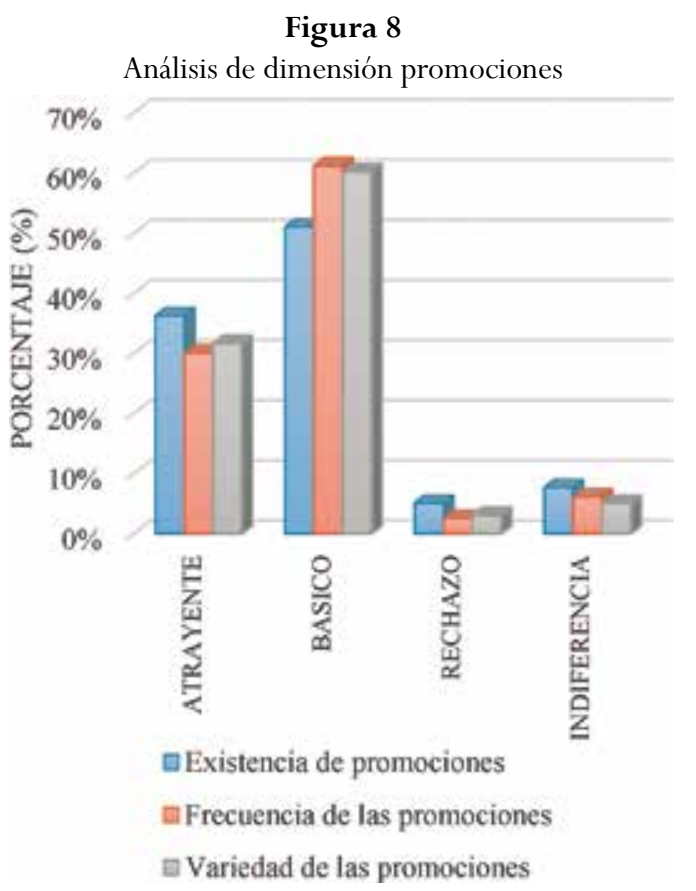

\section{Dimensión: Rapidez}

En esta dimensión, se incluye un atributo que se considera importante en toda relación de compra venta, el cual está referido al tiempo de espera del cliente, el mismo que más valora. Del total de los encuestados 112 consideran como atrayente, mientras que 25 lo considera como básico y 50 lo considera como factor de rechazo y solamente 8 lo considera como indiferente.

Estas respuestas se expresan en términos porcentuales; tal es así que, el 57.1\% lo considera como atra- yente; mientras $25.5 \%$ lo considera como factor de rechazo y el $13.3 \%$ lo considera como básico y 4.1 $\%$ es indiferente frente a este atributo (Tabla 10). El análisis efectuado a este atributo se observa en la Figura 9.

Tabla 10

Cuadro resumen de respuestas sobre atributos de rapidez

\begin{tabular}{|c|c|c|c|c|c|}
\hline \multirow[b]{2}{*}{ Atributo } & \multicolumn{4}{|c|}{ Respuestas } & \multirow[b]{2}{*}{$\%$} \\
\hline & 苞 & 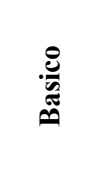 & 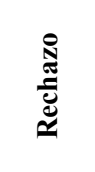 & 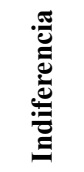 & \\
\hline $\begin{array}{l}\text { Tiempo de espera } \\
\text { del cliente }\end{array}$ & $57.1 \%$ & $13.3 \%$ & $25.5 \%$ & $4.1 \%$ & 100.0 \\
\hline
\end{tabular}

Figura 9

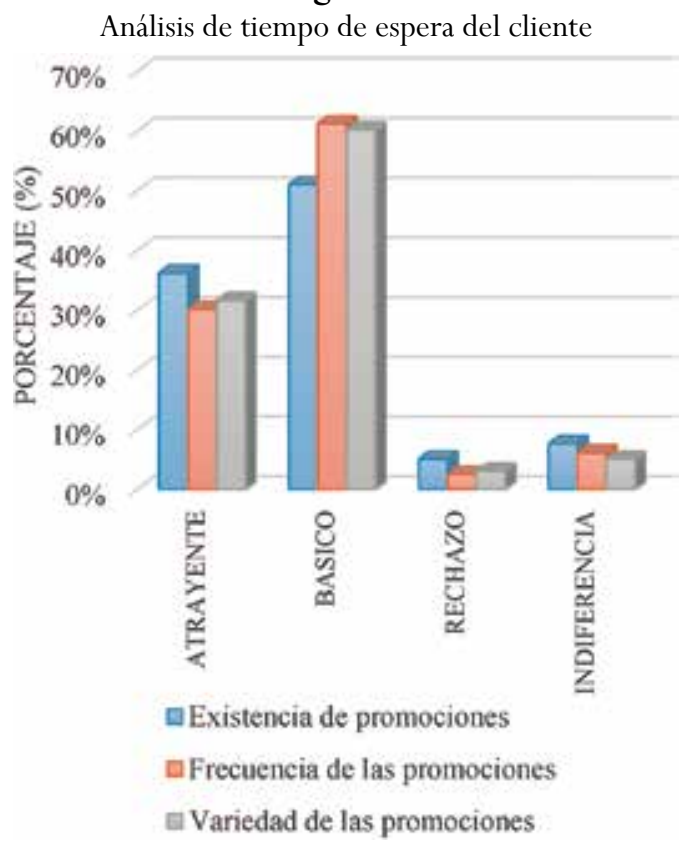

\section{Dimensión: Amabilidad y atención}

En esta dimensión, se ha considerado los atributos de saludo, empatía y gentileza de los empleados, aspectos importantes en la relación con los clientes y, de acuerdo a las respuestas, se tiene que 118 responden como básico a la empatía de los empleados, mientras que 72 señalan que lo atrayente es la gentileza de los empleados; mientras que el de indiferencia es el saludo de los empleados.

En la Tabla 11 , se señala que el $36.7 \%$ considera como atrayente la gentileza de los empleados; mientras que en lo básico, con $60.2 \%$ es considerado como empatía de los empleados; así como el de rechazo con el $4.1 \% \mathrm{y}$; el de indiferencia, el $7.1 \%$ corresponde al saludo de los empleados. El análisis efectuado a los datos referidos a este atributo se puede apreciar en la Figura 10. 
Tabla 11

Cuadro resumen de respuestas sobre atributos de amabilidad y atención

\begin{tabular}{|c|c|c|c|c|c|}
\hline \multirow[b]{2}{*}{ Atributo } & \multicolumn{4}{|c|}{ Respuestas } & \multirow[b]{2}{*}{$\%$} \\
\hline & 总 & 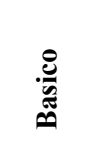 & 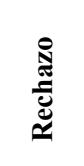 & 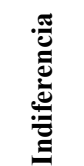 & \\
\hline $\begin{array}{l}\text { Saludo de los em- } \\
\text { pleados }\end{array}$ & $33.2 \%$ & $56.1 \%$ & $3.6 \%$ & $7.1 \%$ & 100.0 \\
\hline $\begin{array}{l}\text { Empatía con los } \\
\text { empleados }\end{array}$ & $29.1 \%$ & $60.2 \%$ & $4.1 \%$ & $6.6 \%$ & 100.0 \\
\hline $\begin{array}{l}\text { Gentileza de los } \\
\text { empleados }\end{array}$ & $36.7 \%$ & $56.1 \%$ & $2.6 \%$ & $4.6 \%$ & 100.0 \\
\hline
\end{tabular}

Figura 10

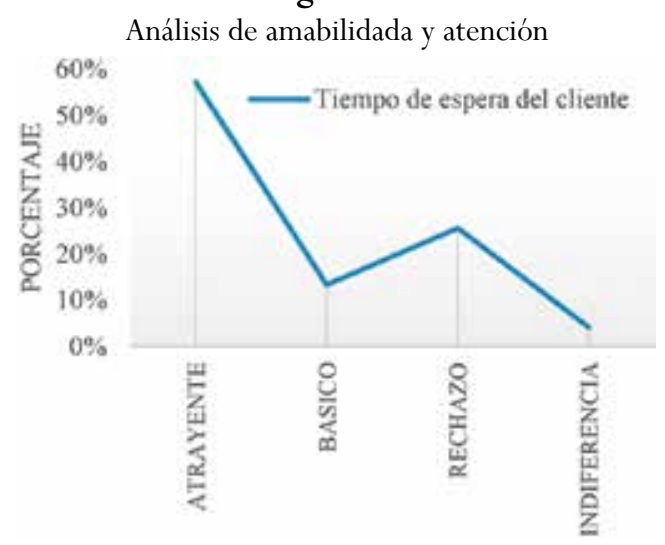

\section{Dimensión: Información}

Esta dimensión es de suma importancia, puesto que los clientes siempre buscan información con la finalidad de tomar una decisión y, también, es una característica de estos tiempos, puesto que el cliente es una persona que dispone de mucha información y en el caso de alimentos siempre están buscando productos que no le afecten a la salud. Dentro de esta dimensión, se ha considerado los atributos de conocimiento del producto de parte de los empleados; la descripción y la rapidez de la entrega de la información. Por ello, es necesario que los empleados, que tengan relación directa con los clientes, deban ser quienes estén bien informados para así responder con prontitud cualquier interrogante que les formulen.

Del total de los encuestados, 59 consideran como atrayente a la rapidez de la entrega de la información; mientras que 109 consideran como básico a la descripción de los productos de la carta y, también 15 consideran a este atributo como de rechazo; mientras 33, responden como indiferentes al conocimiento de los empleados de los productos ofertados por la empresa.

En términos porcentuales; el 30.1\% consideran como atrayente a la rapidez de la entrega de la información; del mismo modo, este atributo es considerado como básico por el $58.7 \%$; de rechazo por el $7.7 \%$ a la descripción de los productos y; el $16.8 \%$, considera como indiferente al conocimiento de los empleados en relación al producto (Tabla 12). Los que se pueden observar en la Figura 11.

\section{Tabla 12}

Cuadro resumen de respuestas sobre atributos de información de los empleados de venta.

\begin{tabular}{llllll}
\hline \multicolumn{1}{c}{ Atributo } & \multicolumn{5}{c}{ Respuestas } \\
\cline { 2 - 5 } & & & & & \\
& & & & & \\
\hline $\begin{array}{l}\text { Conocimiento de } \\
\text { los empleados en } \\
\text { relación al pro- } \\
\text { ducto }\end{array}$ & $26.5 \%$ & $50.5 \%$ & $6.1 \%$ & $16.8 \%$ & 100.0 \\
$\begin{array}{l}\text { Descripción de los } \\
\text { productos de la } \\
\text { carta }\end{array}$ & $24.5 \%$ & $55.6 \%$ & $7.7 \%$ & $12.2 \%$ & 100.0 \\
$\begin{array}{l}\text { Rapidez en la } \\
\text { entrega de la infor- } \\
\text { mación }\end{array}$ & $30.1 \%$ & $58.7 \%$ & $5.1 \%$ & $6.1 \%$ & 100.0 \\
\hline
\end{tabular}

\section{Figura 11}

Análisis de dimensión de información de los empleados de venta

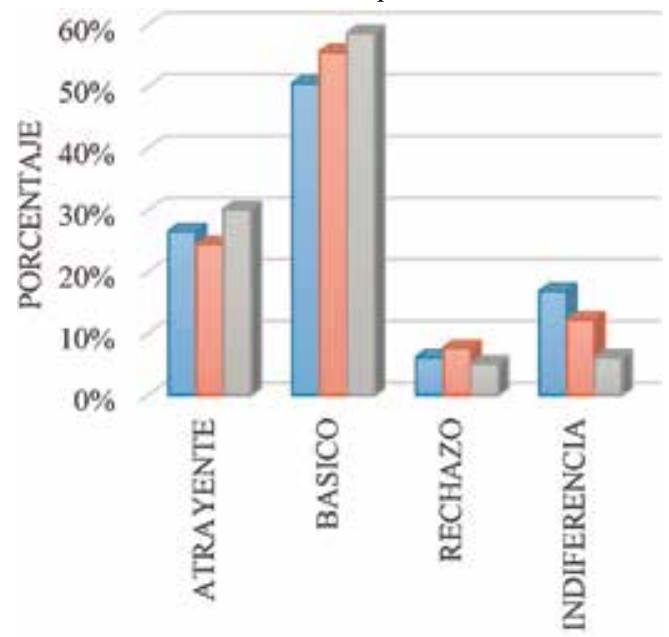

- Conocimiento de los empleados en relación al producto

a Descripción de los productos de la carta

$\square$ Rapidez en la entrega de la información

\section{Dimensión: Ambiente y decoración}

En esta dimensión se considera a los atributos de música, olores y colores agradables; factores a tenerse en cuenta en la presentación del local y que los clientes valoran al visitar un local comercial o simplemente un hogar familiar que le provoque tranquilidad y sosiego y un descanso placentero. 
Del total de los entrevistados; 75 responden como atrayente a la música agradable; 118 consideran como básico a los olores; 8 consideran como de rechazo a los colores y; 7 consideran indiferencia a los olores agradables.

La música agradable es considerada como; atrayente, por el $38.3 \%$; mientras que el $60.2 \%$, lo considera como básico y; el $4.1 \%$ de rechazo a los colores; así como, el de indiferencia con el 3.6\% a los olores agradables (Tabla 13). El que se puede apreciar en la Figura 12.

Tabla 13

Cuadro resumen de respuestas sobre atributos de ambiente y decoración

\begin{tabular}{|c|c|c|c|c|c|}
\hline \multirow[b]{2}{*}{ Atributo } & \multicolumn{4}{|c|}{ Respuestas } & \multirow[b]{2}{*}{$\%$} \\
\hline & 苞 & 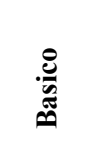 & 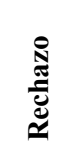 & 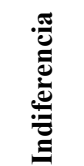 & \\
\hline Música agradable & $38.3 \%$ & $57.1 \%$ & $2.0 \%$ & $2.6 \%$ & 100.0 \\
\hline Olores agradables & $33.2 \%$ & $60.2 \%$ & $3.1 \%$ & $3.6 \%$ & 100.0 \\
\hline Colores agradables & $36.7 \%$ & $58.2 \%$ & $4.1 \%$ & $1.0 \%$ & 100.0 \\
\hline
\end{tabular}

Figura 12

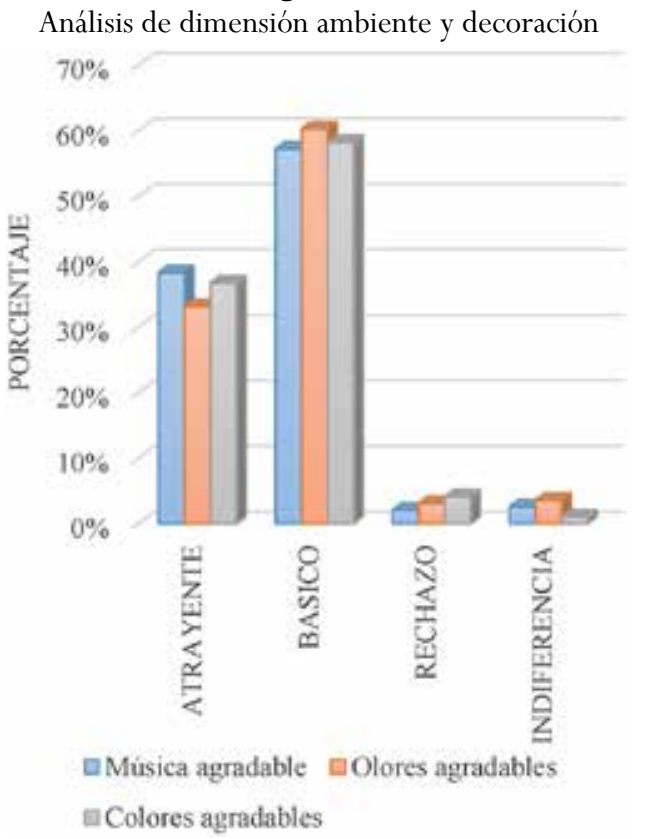

\section{Dimensión: Higiene}

En esta dimensión se consideran los atributos de pisos limpios e higiénicos, la barra limpia, la zona de atención, la presentación del personal, los tazones y utensilios limpios e higiénicos y el contenedor de desperdicios; atributos que el cliente debe apreciar y son los que más valoran en cualquier establecimiento de comida.

En el caso de estudio, se tiene que los tazones limpios son considerados como atrayentes por 104 encuesta- dos, debido a que en este recipiente se presenta las cremas y ensaladas; mientras que 96 , responden como básico a la barra limpia y; de rechazo a la zona de atención y de indiferencia, 16 consideran como presentación del personal.

Porcentualmente; el 53.1\% considera como atrayente a los tazones limpios e higiénicos; $49.0 \%$, como básico; $2.0 \%$, como de rechazo a la zona de atención y; el de indiferencia, con el $8.2 \%$ a la presentación del personal, tal como se puede apreciar en la Tabla 14 y la Figura 13.

Tabla 14

Cuadro resumen de respuestas sobre atributos de higiene

\begin{tabular}{|c|c|c|c|c|c|}
\hline \multirow[b]{2}{*}{ Atributo } & \multicolumn{4}{|c|}{ Respuestas } & \multirow[b]{2}{*}{$\%$} \\
\hline & 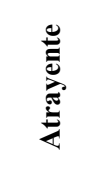 & 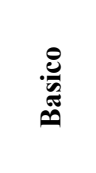 & 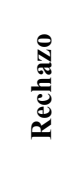 & 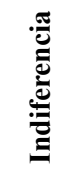 & \\
\hline $\begin{array}{l}\text { Pisos limpios e } \\
\text { higiénicos }\end{array}$ & $46.9 \%$ & $43.9 \%$ & $1.5 \%$ & $7.7 \%$ & 100.0 \\
\hline $\begin{array}{l}\text { Barra limpias e } \\
\text { higiénicas }\end{array}$ & $44.4 \%$ & $49.0 \%$ & $1.0 \%$ & $5.6 \%$ & 100.0 \\
\hline $\begin{array}{l}\text { Zona de atención } \\
\text { cómoda y amplia }\end{array}$ & $47.4 \%$ & $45.4 \%$ & $2.0 \%$ & $5.1 \%$ & 100.0 \\
\hline $\begin{array}{l}\text { Presentación per- } \\
\text { sonal de aseo }\end{array}$ & $48.5 \%$ & $41.8 \%$ & $1.5 \%$ & $8.2 \%$ & 100.0 \\
\hline $\begin{array}{l}\text { Tazones limpias } \\
\text { e higiénicas }\end{array}$ & $53.1 \%$ & $44.4 \%$ & $1.0 \%$ & $1.5 \%$ & 100.0 \\
\hline $\begin{array}{l}\text { Contenedor de } \\
\text { desperdicios }\end{array}$ & $51.5 \%$ & $43.4 \%$ & $1.0 \%$ & $4.1 \%$ & 100.0 \\
\hline
\end{tabular}

Figura 13

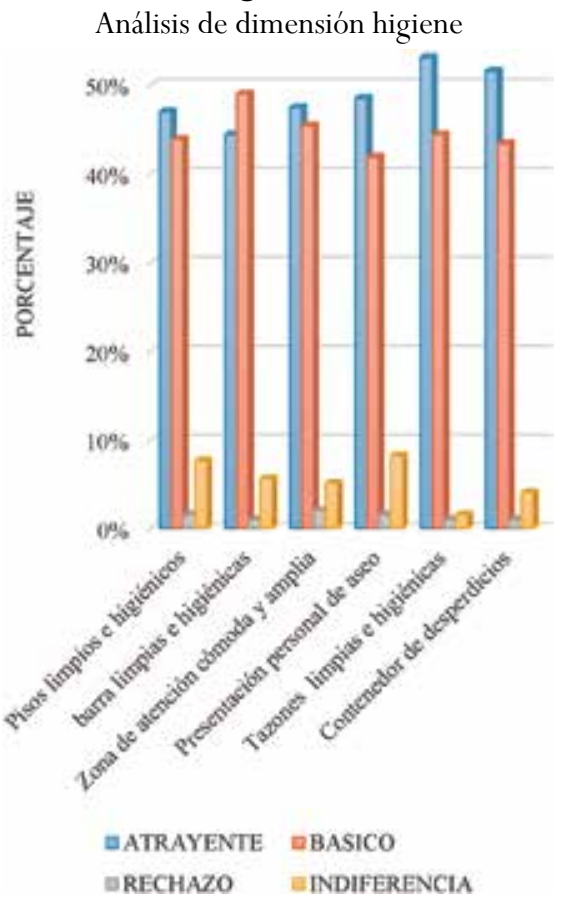




\section{Discusión}

Para determinar la incidencia de la satisfacción del cliente en la rentabilidad, se ha utilizado el SPSS 22 el cual se ha aplicado a los datos obtenidos a través de la encuesta realizada a la muestra determinada de 196 clientes, lo que ha permitido determinar, cómo el nivel de satisfacción de clientes influye en forma moderada y positiva en la rentabilidad. Esta influencia se explica debido a que la satisfacción de clientes influye en el retorno a sus proveedores de bienes o servicios, pero se debe tener en cuenta que, si bien es cierto que el grado de satisfacción es moderada o alta, el cliente sigue siendo independiente al tomar decisiones de compra, pudiendo elegir o no elegir el mismo establecimiento pese a que se haya logrado tener una mayor satisfacción.

El modelo Kano, ha permitido establecer los grados de satisfacción, al lograr establecer los diversos conceptos de calidad en la cual se ha diferenciado los diversos atributos que el cliente valora y entendemos que no es en la misma proporción.

\section{Contrastación de la hipótesis}

\section{Hipótesis general}

El modelo Kano de satisfacción de clientes, influye positivamente en la rentabilidad de las Mypes de la ciudad de Huancayo.

En esta hipótesis, se tiene la variable independiente satisfacción del cliente y la variable dependiente rentabilidad, la que se determina empleando el sistema Dupont y lo que se quiere probar es la influencia de la variable satisfacción del cliente en la variable dependiente rentabilidad; para ello, se realizó los siguientes pasos:

\section{Plantear la hipótesis nula y alterna}

\section{Hipótesis nula:}

HO = El modelo Kano de satisfacción de clientes, influye negativamente en la rentabilidad de las Mypes de la ciudad de Huancayo.

\section{Hipótesis Alterna:}

H1 = El modelo Kano de satisfacción de clientes, influye positivamente en la rentabilidad de las Mypes de la ciudad de Huancayo.

Plantear el nivel de significancia; se establece el margen de error de la prueba.

$$
\&=0.05 \text { donde Alfa }=0.05
$$

Se plantea el valor de prueba

$$
\text { Spearman }=0.545
$$

Comparación de p y \&

$$
\mathrm{p} \text { valor }=0.000<\&=0.05
$$

Para la comparación se tiene en cuenta la regla de Spearman, que se ve en el siguiente cuadro:

\begin{tabular}{l}
$\frac{\text { Regla para Spearman }}{\text { de } 0.0 \text { a } 0.2=\text { Muy baja o muy débil }}$ \\
de 0.2 a $0.4=$ Baja o débil \\
de 0.4 a $0.6=$ Moderada \\
de 0.6 a $0.8=$ Alta o fuerte \\
de 0.8 a $1.0=$ Muy alta o muy fuerte \\
\hline
\end{tabular}

\section{Decisión}

De acuerdo a la comparación efectuada tendríamos que rechazar la hipótesis nula y nos quedaría la hipótesis alterna.

\section{Conclusión}

Para la conclusión, se planteó la hipótesis alterna, indicando lo siguiente: "El modelo Kano de satisfacción de clientes, influye de manera lineal moderadamente directa y positivamente en la rentabilidad de las Mypes de la ciudad de Huancayo.”

\section{Hipótesis Especifica 1}

La implementación del modelo Kano de satisfacción de clientes, influye en el incremento de las ventas netas de las Mypes de la ciudad de Huancayo.

En esta hipótesis, se tiene la variable independiente satisfacción del cliente y la variable dependiente ventas netas y; lo que se quiere probar es la influencia de la variable satisfacción del cliente en la variable dependiente ventas; para ello, se realizaron los siguientes pasos:

\section{Plantear la Hipótesis nula y alterna}

\section{Hipótesis nula:}

H0 = La implementación del modelo Kano de satisfacción de clientes, no influye en el incremento de las ventas netas de las Mypes de la ciudad de Huancayo.

\section{Hipótesis Alterna:}

H1 = La implementación del modelo Kano de Satisfacción de clientes influye en el incremento de las ventas netas de las Mypes de la ciudad de Huancayo.

Plantear el nivel de significancia; se establece el margen de error de la prueba.

$$
\&=0.05 \text { donde Alfa }=0.05
$$

Se plantea el valor de prueba

$$
\text { Spearman }=0.467
$$

Comparación de p y \&

$$
\mathrm{p} \text { valor }=0.000<\&=0.05
$$

Para la comparación se tiene en cuenta la regla de Spearman. 


\section{Decisión}

De acuerdo a la comparación efectuada, se tendría que rechazar la hipótesis nula y quedaría la hipótesis alterna.

\section{Conclusión}

Para la conclusión, se plantea la hipótesis alterna, indicando lo siguiente: "La implementación del modelo Kano de satisfacción de clientes, influye moderadamente directa en el incremento de las ventas netas de las Mypes de la ciudad de Huancayo."

\section{Hipótesis Específica 2}

El modelo Kano de satisfacción del cliente influye positivamente en la solvencia financiera de las mypes de la ciudad de Huancayo.

En esta hipótesis, se tiene la variable independiente satisfacción del cliente y la variable dependiente: solvencia financiera y lo que se quiere probar es la influencia de la variable satisfacción del cliente en la variable dependiente solvencia financiera, para ello, se realizaron los siguientes pasos:

\section{Plantear la Hipótesis nula y alterna}

\section{Hipótesis nula:}

$\mathrm{HO}=$ El modelo Kano de satisfacción del cliente, no influye positivamente en la solvencia financiera de las Mypes de la ciudad de Huancayo.

\section{Hipótesis Alterna:}

H1 = El modelo Kano de satisfacción del cliente, influye positivamente en la solvencia financiera de las Mypes de la ciudad de Huancayo.

Plantear el nivel de significancia; se establece el margen de error de la prueba.

$$
\&=0.05 \text { donde Alfa }=0.05
$$

Se plantea el valor de prueba

$$
\text { Spearman }=0.448
$$

Comparación de p y \&

$$
\mathrm{p} \text { valor }=0.000<\&=0.05
$$

Para la comparación se tiene que tener en cuenta la regla de Spearman.

\section{Decisión}

De acuerdo a la comparación efectuada, se tendría que rechazar la hipótesis nula y quedaría la hipótesis alterna.

\section{Conclusión}

Para la conclusión, se plantea la hipótesis alterna indicando lo siguiente: "El modelo Kano de satisfacción del cliente, influye moderadamente directo y positivamente en la solvencia financiera de las Mypes de la ciudad de Huancayo."

\section{Conclusiones}

- El modelo Kano, se constituye en una herramienta esencial en la determinación del nivel de satisfacción del cliente; concepto que difícilmente se puede definir, puesto que ello está en función a la percepción del cliente, mas no de la empresa. Por esta razón, este modelo permite afrontar con éxito y definir este concepto, debido a que en base a ella se establece la lealtad del cliente y, por ende, la retención del cliente, el cual, luego de haberse aplicado la prueba de Spearman, se ha establecido la relación directa positiva de la variable satisfacción de clientes con la rentabilidad y, se debe de tener presente que este resultado puede inferir a las empresas de la ciudad de Huancayo, consideradas como Mypes.

- El nivel de satisfacción de clientes influye en forma moderada y positiva en el incremento de las ventas y, por ende, en la solvencia financiera de la empresa, toda vez que cuando existe una mayor satisfacción, vamos a tener clientes más leales a la empresa y que su permanencia en la organización está garantizada y va a formar parte de la organización y en algunas ocasiones manifestaran su adhesión y alguna recomendación para mejorar el producto o el servicio de atención.

\section{Referencias bibliográficas}

Buj López, A. (2015). http://190.242.62.234:8080/ jspui/ bitstream/11227/3983/1/ Estudio comprativo de la satsfacción del cliente.

Cahuaya Rivera Rocío y Ñahuincopa Arango Pablo. (2016). repositorio.uncp.edu.pe/ bitstream/.../ TESIS\%20 Cahuaya -Ñahuincopa.pdf.

Enrique, V. M. (2014). http: / / tesis.pucp.edu.pe/ repositorio/handle/123456789/5536.

Española, R. A. (s.f.). https://definicion.de /rentabilidad.

Gitman, Lawrence J. y Zutter, Chad J. Principios de administración financiera, Decimocuarta edición, Editorial Pearson, México 2016.

Herrerías, Rafael; Palacios Federico y Callejón José; Técnicas cuantitativas para la inferencia, Delta $\mathrm{Pu}$ blicaciones, Madrid España 2012.

Johanna, S. S. (2018). biblioteca.universia.net/.../params/ .../evaluacion-modelo-medicion -impacto -estrategia.

León Chinchilla, Anabelle y Verela Fallas, Maribel. (2011). La rentabilidad. Revista de Ciencias Económicas, $\mathrm{N}^{\circ} 01$ Volumen 29.

Rey, Ana M. y Silvestre; Alejandro A. Manual de higiene alimentaria para manipuladores y consumidores; AMV Ediciones, Madrid, España 2016. 